\title{
Impact of spatio-temporal thermal decoherence on soliton microcombs in multimode microresonators
}

\author{
Qi-Fan Yang ${ }^{1, \dagger}$, Qing-Xin $\mathrm{Ji}^{1,2, \dagger}$, Lue $\mathrm{Wu}^{1, \dagger}$, Boqiang Shen ${ }^{1}$, Heming Wang ${ }^{1}$, \\ Zhiquan Yuan ${ }^{1}$, Chengying Bao ${ }^{1}$, Maodong Gao ${ }^{1}$, and Kerry Vahala ${ }^{1, *}$ \\ ${ }^{1}$ T. J. Watson Laboratory of Applied Physics, California Institute of Technology, Pasadena, California 91125 , \\ USA \\ ${ }^{2}$ School of Physics, Peking University, Beijing 100871 PRC \\ $\dagger$ These authors contributed equally to this work. \\ *vahala@caltech.edu
}

\begin{abstract}
The phase noise of the soliton repetition rate is experimentally characterized in silica microresonators. In conjunction with dispersive wave quieting of pump technical noise, spatio-temporal fluctuations of distinct transverse modes set a limit to performance. ㄷ 2020 The Author(s)
\end{abstract}

OCIS codes: (190.4360) Nonlinear optics, devices; (140.3945) Microcavities; (190.5530) Pulse propagation and temporal solitons.

The demonstration of soliton mode-locking in optical microresonators has given rise to a new class of miniaturized optical frequency combs that enable chip-based applications such as optical frequency synthesis and optical clocks [1]. The stability of the soliton repetition rate is important across a wide range of applications, and also when the comb functions as a stand-alone microwave signal source. While the intrinsic noise set by quantumlimited motion of the solitons is very low [2], technical noise contributions are found to dominate. For example phase noise in the optical pump can couple to the soliton repetition rate through a process driven by the Raman self-frequency shift [3]. An effective method to suppress this coupling is by operation at a so-called quiet point formed when a strong single-mode dispersive wave is emitted by the soliton [4]. Dispersive waves are generated when a mode of the soliton forming mode becomes degenerate with a mode belonging to another mode family. The spectral recoil from their interaction can be tuned to balance the Raman effect and thereby decouple the pump noise at a specific operating point. Here we show that the suppression possible using this mechanism can be very large. Moreover, we identify a fundamental limit set by spatio-thermal fluctuations of the two interacting modes.

Soliton microcombs are generated in an ultra-high-Q silica microresonator with $15.2 \mathrm{GHz}$ free-spectral-range (optical spectrum in Fig. 1(a)) [3]. Significantly, the spectrum features a strong dispersive wave that is used below for quiet point operation. The microresonator is sealed in a metal package and mounted in an acoustic shield for mechanical and thermal isolation. Photodetection of the soliton microcombs reveals high-contrast, narrowlinewidth beatnotes as shown in Fig. 1(b). Figure 1(c) gives the relative Allan deviation of the beatnote, which reaches $5 \times 10^{-11}$ at $40 \mathrm{~ms}$ integration time with long-term drift less than $0.1 \mathrm{~Hz} / \mathrm{s}$.
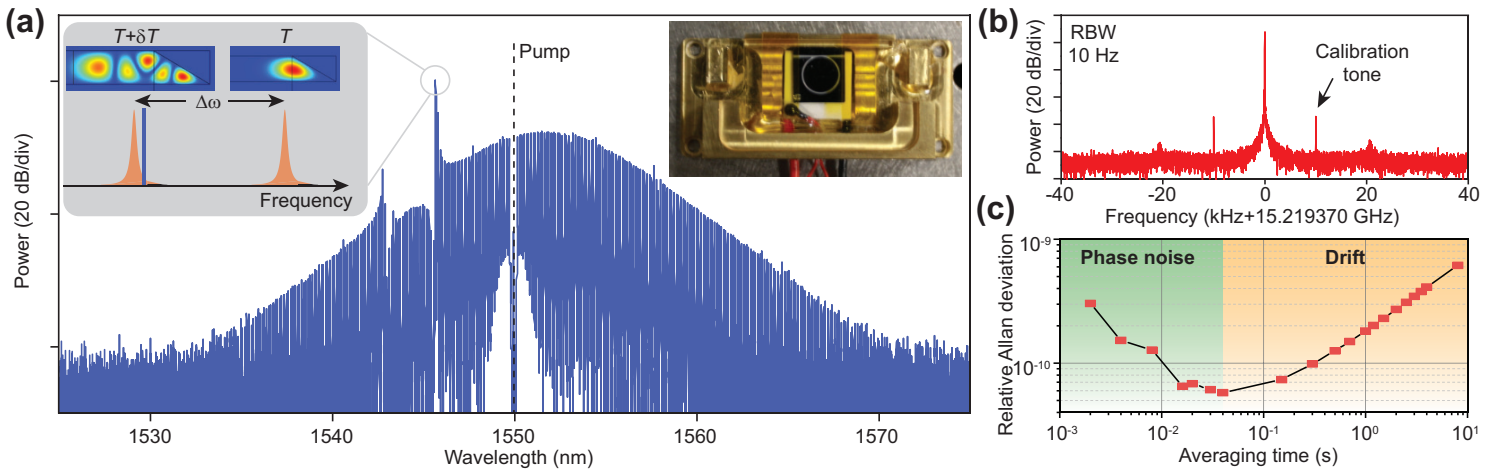

Fig. 1. Optical and electrical spectra of soliton microcomb. (a) Optical spectrum of the soliton microcomb. The pump (dashed line) and the highlighted dispersive wave belong to fundamental and higher-order mode families, respectively. Inset right: silica microresonator in a package. Inset left: spatio-temporal thermal tuning of soliton and dispersive wave modes. (b) Electrical spectrum showing soliton repetition rate. (c) Allan deviation of repetition rate. 

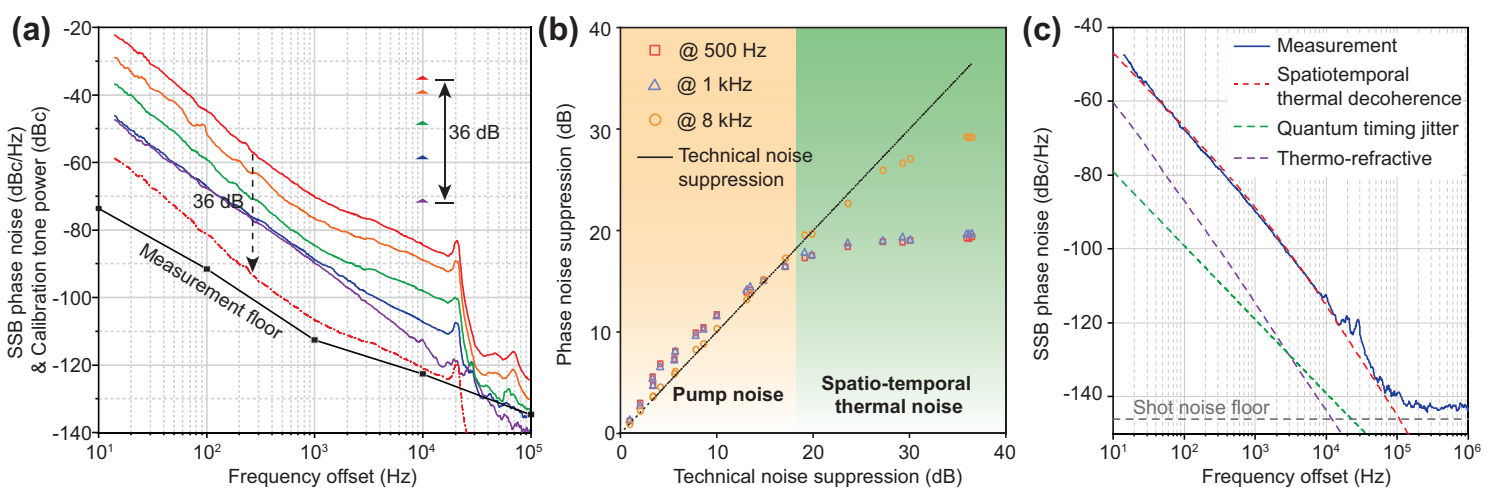

Fig. 2. Quiet point and spatio-temporal thermal decoherence. (a) Phase noise and calibration tone power at different detuning (indicated by color). Pump contributed noise corresponding to the weakest calibration tone is also plotted (dashed line). (b) Plot of actual noise versus calibration tone suppression at several offset frequencies. (c) Measured phase noise at maximum quiet point suppression and calculated spatio-temporal thermal decoherence noise. Quantum timing jitter, thermorefractive noise and detector shot noise are also plotted.

Quiet-point operation is established as described in Ref. [4]. Plotted in Fig. 2(a) are phase noise traces of the repetition rate while approaching the quiet point. Tuning control in this process is possible by adjusting the detuning of the pump frequency relative to the cavity resonance that is pumped. In addition, calibration tones are created by modulating the phase of the pump laser at $10 \mathrm{kHz}$. This enables active monitoring of the degree to which pump phase noise is suppressed. At the quiet point $36 \mathrm{~dB}$ noise suppression is achieved, which is sufficient to reduce the pump noise below the measured phase noise. Further comparison between the amount of reduction in pump noise (as inferred by the tone) and measured phase noise reveals a regime dominated by another source of noise (Fig. 2(b)).

Noise contributions to the repetition rate are calculated in Fig. 2(c), including quantum timing jitter [2], thermorefractive noise [5] and detector shot noise. And each source is too small to be a limiting factor in this work. A new source of noise caused by spatio-temporal thermal decoherence is believed to set the observed noise limit. Due to their spatial displacement, the soliton spatial mode and the transverse mode associated with the dispersive wave are not in thermal equilibrium. Moreover, as illustrated in Fig. 1(a), their temperature difference $\delta T$ changes their frequency difference $\Delta \omega$, thereby altering the power of the dispersive wave and in turn the repetition rate [4]. The contribution of this spatio-temporal thermal fluctuation to the noise of soliton repetition rate can be shown to be given by $S_{\mathrm{ST}}=\left(\alpha^{2} n_{T}^{2} \omega^{2} / n_{o}^{2}\right) S_{\delta T}$. Here, $n_{T}$ and $n_{o}$ are the thermo-optic coefficient and the refractive index, respectively; $\omega$ is the frequency of the dispersive wave and $\alpha=\partial \omega_{r} / \partial \Delta \omega$ is the dependence of the repetition rate $\omega_{r}$ on $\Delta \omega$. The power spectral density $S_{\delta T}$ can be obtained numerically using the finite-element-method [5]. The resulting calculated $S_{\mathrm{ST}}$ (Fig. 2(c)) is in good agreement with the measurement. In making this plot the coefficient $\alpha$ is a fitting parameter and the fit value of $-26 \mathrm{~dB}$ is close to the value $-22 \mathrm{~dB}$ derived from measured soliton properties based on the theory in Ref. [4].

In summary, we have studied the quiet point operation of a soliton microcomb for improved repetition rate stability. Spatio-temporal thermal fluctuations are believed to present a limit on the stability at the quiet point. The quiet point suppression mechanism, itself, was measured to provide up to $36 \mathrm{~dB}$ of pump-noise suppression.

\section{References}

1. T. J. Kippenberg, A. L. Gaeta, M. Lipson, and M. L. Gorodetsky, "Dissipative Kerr solitons in optical microresonators," Science 361 (2018).

2. A. B. Matsko and L. Maleki, "On timing jitter of mode locked Kerr frequency combs," Opt. Express 21, 28862-28876 (2013).

3. X. Yi, Q.-F. Yang, K. Y. Yang, M.-G. Suh, and K. Vahala, "Soliton frequency comb at microwave rates in a high-Q silica microresonator," Optica 2, 1078-1085 (2015).

4. X. Yi, Q.-F. Yang, X. Zhang, K. Y. Yang, X. Li, and K. Vahala, "Single-mode dispersive waves and soliton microcomb dynamics," Nat. Commun. 8, 14869 (2017).

5. N. Kondratiev and M. Gorodetsky, "Thermorefractive noise in whispering gallery mode microresonators: Analytical results and numerical simulation,” Phys. Lett. A 382, 2265-2268 (2018). 